\title{
EPÍFITAS VASCULARES DA VILA DOIS RIOS, ILHA GRANDE, RJ: ASPECTOS ECOLÓGICOS, ENDEMISMOS E CONSERVAÇÃO ${ }^{1}$
}

\author{
VASCULAR EPIPHYTES OF VILA DOIS RIOS, ILHA GRANDE, RJ: ECOLOGICAL \\ ASPECTS, ENDEMISMS AND CONSERVATION
}

\section{Ana Carolina Rodrigues da $\mathrm{Cruz}^{2,3, *}$, Nadjara de Medeiros Corrêa ${ }^{4}$ 5, André Felippe Nunes-Freitas ${ }^{4,6}$ \& Lana da Silva Sylvestre ${ }^{7}$}

\begin{abstract}
${ }^{2}$ Programa de Pós-graduação em Botânica, Museu Nacional, Horto Botânico - Quinta da Boa Vista s.n., São Cristóvão, CEP 20940-040, Rio de Janeiro, RJ, Brasil; ${ }^{3}$ ORCID: 00000002-4041-0384; ${ }^{4}$ Universidade Federal Rural do Rio de Janeiro, Instituto de Florestas, BR-465, Km 7, CEP 23897-000, Seropédica, RJ, Brasil; ${ }^{5}$ ORCID: 0000-0002-2378-2936; ${ }^{6}$ ORCID: 0000-0002-5149-9070; ${ }^{7}$ Universidade Federal do Rio de Janeiro, CCS/IB, Ilha do Fundão, CEP 21941-599, Rio de Janeiro, RJ, Brasil; ${ }^{8}$ ORCID: 0000-0002-0486-0898; *Autor de correspondência: anacruzufrj@gmail.com
\end{abstract}

\begin{abstract}
Resumo: As plantas epífitas representam um dos grupos mais ricos e complexos dos neotrópicos e possuem grande importância ecológica nos ambientes. Árvores isoladas em áreas rurais ou urbanas abrigam comunidades epifíticas bastante diversas, porém poucos trabalhos tem abordado essa questão. Diante disso, o objetivo deste estudo foi inventariar a riqueza e composição de epífitas vasculares da Vila Dois Rios e descrever aspectos ecológicos, de distribuição geográfica e estado de conservação das espécies. Registramos as epífitas ocorrentes nas praças e avenidas da Vila Dois Rios, Ilha Grande, RJ, e em florestas próximas em diferentes estágios de regeneração. Foram inventariadas 85 espécies, pertencentes a 40 gêneros e 15 famílias. Bromeliaceae e Polypodiaceae foram as famílias com maior riqueza. A maioria é holoepífita, entomófila, anemocórica e endêmica da Floresta Atlântica. Três espécies são restritas à Região Sudeste e nenhuma encontra-se ameaçada de extinção, porém $73,8 \%$ não foram avaliadas quanto ao grau de ameaça. Nossos resultados reforçam que a Ilha Grande é uma importante área de conservação da biodiversidade e que a arborização urbana tem um importante papel na manutenção da comunidade epifítica. Pesquisas ecológicas populacionais sobre essas espécies devem ser priorizadas, pois são fundamentais para a elaboração de estratégias específicas de conservação.
\end{abstract}

Palavras-chave: áreas protegidas; biodiversidade; dossel; epifitismo; Floresta Atlântica.

\begin{abstract}
Epiphyte plants represent one of the richest and most complex groups of neotropics and have great ecological importance in the environment. Isolated trees in rural or urban areas have very diverse epiphyte communities, but there is little research on this. Therefore, the aim of this study was to carry the richness and composition of vascular epiphytes of Vila Dois Rios and describe ecological aspects, geographic distribution and conservation status of the species. We recorded the epiphytes occurring in the squares and avenues of Vila Dois Rios, Ilha Grande, RJ, and in nearby forests at different stages of regeneration. We found 85 species, from 40 genera and 15 families. Bromeliaceae and Polypodiaceae were the families with the greatest richness. Most are holoepiphyte, entomophilous, anemochoric, and endemic to the Atlantic Forest. Three
\end{abstract}

1 Editor de área: Michaele Alvim Milward de Azevedo | Edição: Michaele Alvim Milwardde-Azevedo | Recebido em 16 de março de 2021 | Aceito em 26 de abril de 2021 | Publicado em 20 de julho de 2021. 
species are restricted to the Southeast Region and none are threatened with extinction, however, $73.8 \%$ have not been assessed as to the degree of threat. Our results reinforce that Ilha Grande is an important biodiversity conservation area and that urban trees play an important role in maintaining the epiphytic community. Population ecological research on these species should be prioritized, as they are fundamental for the elaboration of specific conservation strategies.

Keywords: Atlantic forest; biodiversity; canopy; epiphytism; protected areas.

\section{Introdução}

Epífitas são plantas que germinam e enraízam de modo não parasítico em outras plantas em alguma fase da vida (Zotz 2016). Segundo Moffett (2000), as epífitas podem viver em qualquer superfície de outra planta que esteja acima do solo e qualquer efeito negativo que possa causar é indireto, devido ao aumento de peso sobre os galhos. Existem aproximadamente 28.000 espécies de plantas que podem ocorrer como epífitas, o que é equivalente a $9 \%$ das plantas vasculares do planeta (Zotz 2016). As epífitas correspondem a um dos grupos mais diversos e complexos da biodiversidade nos neotrópicos (Gentry \& Dodson 1987) e a Floresta Atlântica, especificamente, é centro de diversidade para o epifitismo, uma vez que possui elevada riqueza e taxa de endemismos (Nieder et al. 1999, 2001, Menini-Neto et al. 2016). De acordo com Ramos et al. (2019), existem mais de 2.000 espécies de plantas epífitas vasculares nas florestas tropicais e subtropicais do Domínio Atlântico incluindo os países Brasil, Argentina, Uruguai e Paraguai, estas informações são corroboradas com os dados recentes presentes na Flora do Brasil 2020.

A comunidade epifítica possui elevada importância nos ecossistemas, pois elas afetam o ciclo hidrológico das florestas através da interceptação da água das chuvas, armazenamento e evapotranspiração das plantas (Holwerda et al. 2013). Epífitas contribuem para a diversificação de microhabitats e nichos ecológicos no dossel florestal, aumentam a disponibilidade de espaço físico, umidade e alimentos disponíveis para a fauna, além de servirem como refúgio reprodutivo para muitos animais (Benzing 1986, Zotz 2016). Bromélias-tanque, por exemplo, acumulam água em seu interior e representam microhabitat para diversas espécies de seres vivos, estes locais constituem a fitotelmata (Zotz 2016). Os habitats de fitotelmata formam ambientes complexos com associações específicas com protozoários, invertebrados e vertebrados e, assim, as bromélias podem funcionar como ampliadoras da biodiversidade, favorecendo o estabelecimento de vários organismos nos ambientes onde ocorrem (sensu Rocha et al. 2000).

As epífitas possuem forte ligação com a umidade atmosférica, pluviosidade e temperatura, por isso são amplamente distribuídas nas florestas tropicais (Madison 1977, Gentry \& Dodson 1987). Em escalas regional e local, a ocorrência das epífitas está relacionada às condições microclimáticas que podem estar associadas ao estágio sucessional das florestas ou ao tipo de uso da terra, dos quais destacamos as 
comunidades que ocorrem em árvores isoladas seja em ambientes rurais ou urbanos (Zotz 2016, Padilha et al. 2017, Richards et al. 2020). Estudos recentes têm mostrado que tais ambientes abrigam ricas e diversificadas comunidades epifíticas com importante papel para conservação das espécies e manutenção do fluxo gênico entre as populações (Alves et al. 2014, Devens et al. 2015, Furtado \& Neto 2015, Santana et al. 2017, Amici et al. 2020, Richards et al. 2020).

A Ilha Grande, município de Angra dos Reis, é um dos mais importantes remanescentes de Floresta Atlântica do estado do Rio de Janeiro, devido ao bom estado de conservação de suas florestas e elevada biodiversidade (Rocha et al. 2003). As pesquisas mostram que há elevada riqueza de plantas epífitas na ilha (Araújo \& Oliveira 1988, Nunes-Freitas et al. 2009, Cruz \& Nunes-Freitas 2017, Cruz \& Nunes-Freitas 2019), entretanto não há estudos publicados sobre as epífitas vasculares ocorrentes em ambientes urbanos e antropizados. Assim, diante da importância taxonômica e ecológica das epífitas para os ambientes e desta lacuna de conhecimento para a ilha, o objetivo deste estudo foi inventariar a riqueza e composição de epífitas vasculares em uma área urbana e em florestas secundárias na Ilha Grande e descrever aspectos ecológicos, de distribuição geográfica e estado de conservação das espécies.

\section{Material e Métodos}

Área de Estudo - a área urbana e florestas amostradas neste estudo localizam-se na Ilha Grande, município de Angra dos Reis no sul do estado do Rio de Janeiro. A ilha possui 65.258 hectares de área com as

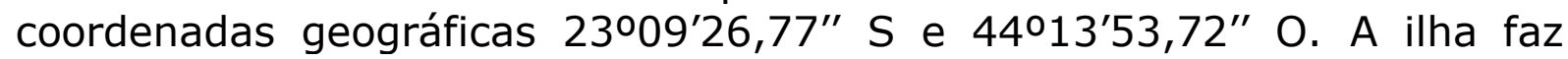
parte de um dos maciços costeiros da Serra do mar e apresenta relevo montanhoso orientado na direção nordeste-sudoeste, sendo que a maior parte do território se situa abaixo de 500 metros de altitude (Gama et al. 2009). Os solos predominantes são do tipo Cambissolos e Espodossolos e o clima é tropical úmido Af de Köppen, quente e úmido, sem estação seca (INEA 2011). A vegetação da ilha é formada por formações diversas associadas ao Domínio Atlântico (Callado et al. 2009), predominando Floresta Ombrófila Densa Submontana e Montana, mas ainda ocorrem Floresta Ombrófila Densa de Terras Baixas, áreas de restingas, matas alagadiças, manguezais e vegetações em afloramentos rochosos (Oliveira $\&$ Coelho Neto 2000).

A área urbana amostrada faz parte da Vila Dois Rios, na vertente oceânica da ilha, e corresponde a um pequeno povoado formado majoritariamente por funcionários do Centro de Estudos Ambientais e Desenvolvimento Sustentável da Universidade do Estado do Rio de Janeiro (CEADS - UERJ) com cerca de 60 moradores (Bastos \& Callado 2009). A vila é cercada por uma rica biodiversidade, embora as suas paisagens já tivessem sido modificadas por vários tipos de uso da terra ao longo dos ciclos econômicos que passaram pela ilha (Bastos \& Callado 2009). A área urbana possui três avenidas, seis ruas, uma travessa e duas estradas (ou "trilhas"). A arborização apresenta principalmente árvores grandes e 
antigas, como palmeiras imperiais (Roystonea oleracea (Jacq.) O. F. Cook, Arecaceae), mangueiras (Mangifera indica L., Anacardiaceae) e abacateiros (Persea americana Mill., Lauraceae), localizados ao longo das avenidas, ruas e praças, e ainda há a presença de muitas amendoeiras (Terminalia catappa L., Combretaceae) na orla da praia (Bastos \& Callado 2009). Já as áreas florestais amostradas são próximas a vila e correspondem a Floresta Ombrófila Densa de Terras Baixas e Submontana em estágios iniciais e intermediários de regeneração.

Coleta dos Dados - O levantamento das epífitas vasculares foi baseado em amostragens sistemáticas e assistemáticas nos anos de 2019 e 2020. A amostragem sistemática foi realizada em três parcelas permanentes do RAPELD (ver Magnusson et al. 2005). Nas parcelas, todas as árvores com Diâmetro à Altura do Peito maior ou igual a 15 centímetros foram amostradas e tiveram as suas espécies de epífitas registradas. Na amostragem assistemática foram incluídas todas as espécies epífitas registradas nas árvores das principais praças e avenidas da Vila Dois Rios. O material botânico foi coletado com o auxílio de um podão telescópico, também foi utilizada a câmera fotográfica Canon PowerShot SX60 HS com super zoom para visualização e identificação dos espécimes. Todo o material coletado recebeu tratamento de herborização tradicional e será depositado no Herbário do Museu Nacional. Não foram coletados espécimes estéreis e em zonas de altura mais altas nas árvores devido a dificuldades metodológicas. A identificação das espécies foi baseada em literatura utilizando-se chaves de identificação específicas, em estudo de material herborizado e com auxílio de especialistas. Os nomes científicos foram verificados em International Plant Names Index (IPNI) e em Brazil Flora Group (BFG 2018).

As espécies foram classificadas em categorias ecológicas quanto à dependência do substrato em holoepífitas, hemiepífitas e epífitas acidentais, de acordo com as definições de Benzing (1990) e baseadas em observações de campo. As síndromes de polinização das espécies foram baseadas em Real (1983) e Proctor et al. (1996) e de dispersão em Gentry \& Dodson (1987). As informações foram levantadas a partir de características morfológicas das espécies e de consultas a bibliografias específicas, não foram realizadas pesquisas aprofundadas sobre a biologia reprodutiva das espécies. As formas de polinização das angiospermas foram classificadas de acordo com as síndromes florais e categorizadas em: anemofilia, quando ocorre através do vento; entomofilia, por meio de insetos; e ornitofilia, através de aves. Quanto à dispersão de diásporos, as espécies foram classificadas em autocóricas, zoocóricas e anemocóricas. As espécies identificadas a nível específico tiveram a distribuição geográfica e endemismo verificados em Flora do Brasil 2020 e Brazil Flora Group (BFG 2021). Foram consideradas de ampla distribuição geográfica àquelas ocorrentes em três ou mais regiões geopolíticas do Brasil e endêmicas aquelas ocorrentes apenas no Domínio Atlântico. O estado de conservação das espécies está de acordo com o Centro Nacional de Conservação da Flora (CNCFlora 2020). 


\section{Resultados e Discussão}

Foram registradas 85 espécies de epífitas vasculares na Vila Dois Rios, pertencentes a 40 gêneros e 15 famílias, sendo que 64 foram identificadas até nível específico (Tabela 1). As áreas de floresta totalizaram 70 espécies $(82,3 \%)$, enquanto a área urbana apresentou 48 $(56,5 \%)$, sendo que $31(36,5 \%)$ foram comuns aos dois ambientes. A menor riqueza em ambientes urbanos e antropizados ocorre devido às epífitas serem sensíveis às mudanças microclimáticas locais, além de apresentarem crescimento lento, tornando-se um dos grupos mais vulneráveis a alterações das condições ambientais (Hietz 1998). Resultados semelhantes já foram observados em outros estudos (Padmawathe et al. 2004, Hietz 2005), porém a riqueza registrada nesta pesquisa foi superior a encontrada por outros autores em ambientes urbanos (Alves et al. 2014, Devens et al. 2015, Furtado \& Neto 2015, Santana et al. 2017). A maior riqueza epifítica pode estar associada tanto à presença de grandes árvores remanescentes na área urbana quanto à proximidade deste ambiente com as áreas florestais que servem como fontes de propágulos, como foi observado por Richards et al. (2020) em comunidades epifíticas de árvores isoladas.

A comunidade epifítica da área de estudo segue a tendência mundial para as epífitas vasculares com a maioria das espécies pertencente às angiospermas (50 espécies, $58,8 \%$ ) e concentrada em poucas famílias (Zotz 2016). As famílias mais importantes em número de espécies foram Bromeliaceae e Polypodiaceae (18 espécies cada, 21,2\%), seguidas por Orchidaceae (15 espécies, $17,6 \%$ ). Os resultados diferem da maioria dos trabalhos realizados em ecossistemas de Mata Atlântica onde se destaca a família Orchidaceae como a mais rica (Kersten 2010). Entretanto, outros autores também registraram Bromeliaceae e Polypodiaceae como famílias mais ricas em ambientes urbanos (Alves et al. 2014, Devens et al. 2015, Furtado \& Neto 2015, Santana et al. 2017). A distribuição da riqueza nas famílias pode ser modificada em florestas secundárias e áreas antropizadas, conforme observado por Barthlott et al. (2001) e Adhikari et al. (2012), com a perda local de algumas famílias mais sensíveis, tais como Orchidaceae (Dislich \& Mantovani 1998, Adhikari et al. 2012).

Quanto a fidelidade ao substrato, 67 espécies são holoepífitas $(78,8 \%), 13$ são hemiepífitas $(15,3 \%)$ e cinco são epífitas acidentais $(5,9 \%)$. O predomínio das holoepífitas, plantas tipicamente epífitas e com adaptações a este modo de vida, é o esperado em estudos realizados em florestas tropicais e tem sido registrado em outras pesquisas no Brasil e no mundo (Nieder et al. 2000, Staudt et al. 2012, Bataghin et al. 2017). Já o hemiepifitismo, quando as plantas podem se conectar ao solo em algum momento de seu ciclo de vida, está relacionado especialmente às famílias Araceae, gêneros Monstera e Philodendron, e Polypodiaceae, algumas espécies, e são comuns em Florestas de Terras Baixas (Benzing 1990), conforme observado neste estudo. De acordo com a Flora do Brasil 2020, as espécies Adiantum latifolium Lam., Adiantopsis radiata (L.) Fée, 
Anthurium urvilleanum Schott, Neoblechnum brasiliense (Desv.) Gasper \& V.A.O. Dittrich e Selaginella muscosa Spring são plantas terrícolas ou rupícolas, porém foram registradas como holoepífitas em florestas ripárias nas proximidades da Vila Dois Rios. A colonização dos forófitos por espécies que não possuem adaptações ao epifitismo se deve a acidentes dispersivos combinado com a acumulação de húmus e elevada umidade nos troncos das árvores (Guzmán-Marín \& Saldaña 2017).

$\mathrm{Na}$ Vila Dois Rios, a maioria das epífitas é polinizada por insetos (36 espécies, 42,3\%), seguido por aves (18 espécies, $21,2 \%$ ) e três são polinizadas pelo vento $(3,5 \%)$ (Figura 1$)$. A entomofilia ocorre principalmente em Orchidaceae e em Araceae, onde a polinização é realizada por uma ampla gama de insetos polinizadores, já a ornitofilia é a forma mais comum em Bromeliaceae, através de beija-flores (Gentry \& Dodson 1987, Mondragón et al. 2015). As epífitas utilizam formas de atração de polinizadores e redução da competição entre estes bastante variadas, como longos períodos de floração, recompensas florais de alta qualidade, produção de fragrâncias florais e até o mimetismo de insetos nas flores (Ackerman 1986, Mondragón et al. 2015). Nossos resultados corroboram com 0 fato de as angiospermas epífitas terem as suas principais formas de polinização associadas à fauna, especialmente abelhas e aves (Gentry \& Dodson 187, Mondragón et al. 2015), o que reforça importância ecológica da comunidade no dossel fornecendo recursos alimentares aos animais que ali habitam (Dettke et al. 2008).

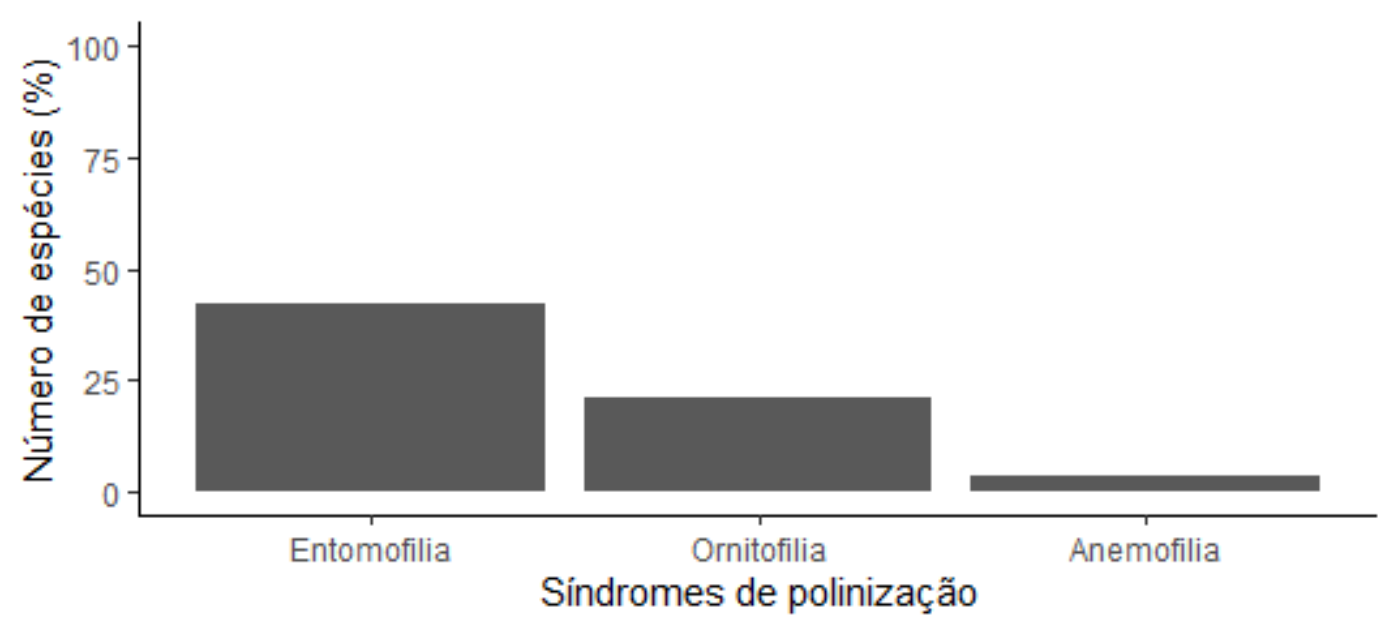

Figura 1. Proporção do número de espécies (\%) de epífitas vasculares por síndromes de polinização da Vila Dois Rios, Ilha Grande, Angra dos Reis, RJ. 
Tabela 1. Lista de espécies de epífitas vasculares ocorrentes em área urbana e florestas secundárias da Ilha Grande, Angra dos Reis, RJ, por família e suas respectivas categorias ecológicas com relação à fidelidade ao substrato, síndromes de polinização e dispersão e estado de conservação, segundo o Centro Nacional de Conservação da Flora (CNCFLORA 2020). CE = categoria ecológica; HOLO = epífita verdadeira; EA = epífita acidental; HEM = hemiepífita; $\mathrm{SP}=$ síndrome de polinização; $E N T$ = entomofilia; ORN = ornitofilia; $\mathrm{ANE}=$ anemofilia; SD = síndrome de dispersão; $Z O O=$ zoocórica; ANEC = anemocórica; $A$ UUTO = autocórica; $N E=$ Não avaliada; $L C=$ Pouco preocupante; $\mathrm{NC}=$ material não coletado.

\begin{tabular}{|c|c|c|c|c|c|c|}
\hline Famílias & Espécies & CE & SP & SD & $\begin{array}{l}\text { Estado de } \\
\text { Conservação }\end{array}$ & $\begin{array}{l}\text { Coletor e } \\
\text { número }\end{array}$ \\
\hline \multirow{10}{*}{ Araceae } & Anthurium scandens (Aubl.) Engl. & HOLO & ENT & ZOO & NE & $\begin{array}{l}\text { ACR Cruz \& NM } \\
\text { Correa } 128\end{array}$ \\
\hline & Anthurium urvilleanum Schott & EA & ENT & ZOO & NE & $\begin{array}{l}\text { ACR Cruz \& NM } \\
\text { Correa } 106\end{array}$ \\
\hline & Epipremnum sp1 & HEM & ENT & $\mathrm{ZOO}$ & - & NC \\
\hline & Monstera adansonii Schott & HEM & ENT & ZOO & NE & $\begin{array}{l}\text { ACR Cruz \& NM } \\
\text { Correa } 100\end{array}$ \\
\hline & Philodendron crassinervium Lindl. & HEM & ENT & ZOO & NE & NC \\
\hline & Philodendron eximium Schott & HEM & ENT & ZOO & NE & $\begin{array}{l}\text { ACR Cruz \& NM } \\
\text { Correa } 103\end{array}$ \\
\hline & Philodendron hastatum K.Koch \& Sello & HEM & ENT & ZOO & NE & $\begin{array}{l}\text { ACR Cruz \& NM } \\
\text { Correa } 88\end{array}$ \\
\hline & Philodendron oblongum (Vell.) Kunth & HEM & ENT & $\mathrm{ZOO}$ & NE & NC \\
\hline & Syngonium podophyllum Schott & HEM & ENT & $\mathrm{ZOO}$ & Exótica & NC \\
\hline & $\begin{array}{l}\text { Thaumatophyllum bipinnatifidum (Schott } \\
\text { ex Endl.) Sakur., Calazans \& Mayo }\end{array}$ & HEM & ENT & ZOO & NE & NC \\
\hline Aspleniaceae & Asplenium auriculatum Sw. & HOLO & - & ANEC & NE & $\begin{array}{l}\text { ACR Cruz et al. } \\
186\end{array}$ \\
\hline Blechnaceae & $\begin{array}{l}\text { Neoblechnum brasiliense (Desv.) Gasper } \\
\text { \& V.A.O. Dittrich }\end{array}$ & EA & - & ANEC & NE & $\begin{array}{l}\text { ACR Cruz \& AF } \\
\text { Nunes-Freitas } \\
53\end{array}$ \\
\hline \multirow[t]{2}{*}{ Bromeliaceae } & Aechmea gracilis Lindm. & HOLO & ORN & ZOO & LC & $\begin{array}{l}\text { ACR Cruz \& NM } \\
\text { Correa } 85\end{array}$ \\
\hline & Aechmea nudicaulis (L.) Griseb. & HOLO & ORN & ZOO & LC & NC \\
\hline
\end{tabular}


Diversidade e Gestão 5: 01-17. 2021

e-ISSN: 2527-0044

http://costalima.ufrrj.br/index.php/diversidadeegestao

\begin{tabular}{|c|c|c|c|c|c|c|}
\hline & Billbergia zebrina (Herb.) Lindl. & HOLO & ORN & $\mathrm{ZOO}$ & LC & NC \\
\hline & $\begin{array}{l}\text { Canistropsis microps (E.Morren ex Mez) } \\
\text { Leme }\end{array}$ & HOLO & ORN & $\mathrm{ZOO}$ & LC & $\begin{array}{l}\text { ACR Cruz et al. } \\
184\end{array}$ \\
\hline & Neoregelia johannis (Carrière) L.B.Sm. & HOLO & ORN & $\mathrm{ZOO}$ & $\mathrm{NE}$ & NC \\
\hline & Tillandsia dura Baker & $\mathrm{HOLO}$ & ORN & ANEC & NE & $\mathrm{NC}$ \\
\hline & Tillandsia gardneri Lindl. & HOLO & ORN & ANEC & LC & $\begin{array}{l}\text { ACR Cruz \& NM } \\
\text { Correa } 126\end{array}$ \\
\hline & Tillandsia geminiflora Brongn & HOLO & ORN & ANEC & LC & $\begin{array}{l}\text { ACR Cruz \& NM } \\
\text { Correa } 150\end{array}$ \\
\hline & Tillandsia stricta Sol. ex Ker Gawl. & $\mathrm{HOLO}$ & ORN & ANEC & NE & NC \\
\hline & Tillandsia tenuifolia L. & HOLO & ORN & ANEC & LC & $\begin{array}{l}\text { ACR Cruz \& NM } \\
\text { Correa } 129\end{array}$ \\
\hline & Tillandsia usneoides (L.) L. & HOLO & ORN & ANEC & LC & $\begin{array}{l}\text { ACR Cruz \& NM } \\
\text { Correa } 131\end{array}$ \\
\hline & Vriesea gigantea Gaudich. & $\mathrm{HOLO}$ & ORN & ANEC & LC & NC \\
\hline & Vriesea lubbersii (Baker) E.Morren & HOLO & ORN & ANEC & LC & $\begin{array}{l}\text { ACR Cruz \& NM } \\
\text { Correa } 120\end{array}$ \\
\hline & Vriesea neoglutinosa Mez & HOLO & ORN & ANEC & LC & NC \\
\hline & Vriesea philippocoburgii Wawra & $\mathrm{HOLO}$ & ORN & ANEC & LC & NC \\
\hline & Vriesea unilateralis (Baker) Mez & HOLO & ORN & ANEC & $\mathrm{NE}$ & NC \\
\hline & Vriesea vagans (L.B.Sm.) L.B.Sm. & HOLO & ORN & ANEC & NE & NC \\
\hline & Vriesea sp1 & $\mathrm{HOLO}$ & ORN & ANEC & - & NC \\
\hline & Lepismium cruciforme (Vell.) Miq. & HOLO & ENT & ZOO & LC & $\begin{array}{l}\text { ACR Cruz \& AF } \\
\text { Nunes-Freitas } \\
68\end{array}$ \\
\hline & Rhipsalis clavata F.A.C.Weber & HOLO & ENT & $\mathrm{ZOO}$ & $\mathrm{NE}$ & $\begin{array}{l}\text { ACR Cruz et al. } \\
153\end{array}$ \\
\hline Cactaceae & Rhipsalis pachyptera Pfeiff. & HOLO & ENT & $\mathrm{ZOO}$ & NE & $\begin{array}{l}\text { ACR Cruz \& NM } \\
\text { Correa } 178\end{array}$ \\
\hline & Rhipsalis teres Steud. & HOLO & ENT & $\mathrm{ZOO}$ & NE & $\begin{array}{l}\text { ACR Cruz \& AF } \\
\text { Nunes-Freitas } \\
58\end{array}$ \\
\hline & Rhipsalis elliptica G.Lindb. ex K.Schum. & HOLO & ENT & $\mathrm{ZOO}$ & LC & ACR Cruz \& NM \\
\hline
\end{tabular}


Diversidade e Gestão 5: 01-17. 2021

e-ISSN: 2527-0044

http://costalima.ufrrj.br/index.php/diversidadeegestao

Correa 114

\begin{tabular}{|c|c|c|c|c|c|c|}
\hline & & \multirow{2}{*}{ NC } \\
\hline & Rhipsalis sp1 & HOLO & ENT & $\mathrm{ZOO}$ & - & \\
\hline \multirow{2}{*}{ Commelinaceae } & Tradescantia fluminensis Vell. & HOLO & ENT & AUTO & NE & $\begin{array}{l}\text { ACR Cruz \& NM } \\
\text { Correa } 127\end{array}$ \\
\hline & Tradescantia zebrina Heynh. ex Bosse & HOLO & ENT & AUTO & $\mathrm{NE}$ & NC \\
\hline Dryopteridaceae & Ctenitis sp1 & HOLO & - & ANEC & - & $\begin{array}{l}\text { ACR Cruz \& NM } \\
\text { Correa } 102\end{array}$ \\
\hline \multirow{2}{*}{ Gesneriaceae } & Codonanthe devosiana Lem. & HOLO & ENT & ZOO & LC & $\begin{array}{l}\text { ACR Cruz \& NM } \\
\text { Correa } 113\end{array}$ \\
\hline & Codonanthe gracilis Hanst. & HOLO & ENT & ZOO & LC & NC \\
\hline \multirow{2}{*}{ Hymenophyllaceae } & Didymoglossum sp1 & HOLO & - & ANEC & - & $\begin{array}{l}\text { ACR Cruz \& NM } \\
\text { Correa } 117\end{array}$ \\
\hline & Didymoglossum sp2 & HOLO & - & ANEC & - & $\begin{array}{l}\text { ACR Cruz \& NM } \\
\text { Correa } 108\end{array}$ \\
\hline Lycopodiaceae & $\begin{array}{l}\text { Phlegmariurus mandiocanus (Raddi) } \\
\text { B.Øllg. }\end{array}$ & HOLO & - & ANEC & NE & $\begin{array}{l}\text { ACR Cruz \& NM } \\
\text { Correa } 133\end{array}$ \\
\hline \multirow{11}{*}{ Orchidaceae } & Acianthera sp1 & HOLO & ENT & ANEC & - & $\begin{array}{l}\text { ACR Cruz \& NM } \\
\text { Correa } 151\end{array}$ \\
\hline & Acianthera sp2 & HOLO & ENT & ANEC & - & $\begin{array}{l}\text { ACR Cruz et al. } \\
188\end{array}$ \\
\hline & Acianthera sp3 & HOLO & ENT & ANEC & - & NC \\
\hline & Cattleya forbesii Lindl. & HOLO & ENT & ANEC & NE & NC \\
\hline & Cattleya sp1 & HOLO & ENT & ANEC & - & NC \\
\hline & Dichaea cogniauxiana Schltr. & HOLO & ENT & ANEC & NE & NC \\
\hline & Encyclia patens Hook. & HOLO & ENT & ANEC & NE & $\begin{array}{l}\text { ACR Cruz \& NM } \\
\text { Correa } 121\end{array}$ \\
\hline & Epidendrum filicaule Lindl. & HOLO & ENT & ANEC & LC & $\begin{array}{l}\text { ACR Cruz \& NM } \\
\text { Correa } 160\end{array}$ \\
\hline & $\begin{array}{l}\text { Epidendrum pseudodifforme Hoehne \& } \\
\text { Schltr. }\end{array}$ & HOLO & ENT & ANEC & NE & NC \\
\hline & $\begin{array}{l}\text { Lankesterella ceracifolia (Barb.Rodr.) } \\
\text { Mansf. }\end{array}$ & HOLO & ENT & ANEC & NE & $\begin{array}{l}\text { ACR Cruz \& NM } \\
\text { Correa } 107\end{array}$ \\
\hline & Lankesterella sp1 & HOLO & ENT & ANEC & - & $\begin{array}{l}\text { ACR Cruz \& NM } \\
\text { Correa } 109\end{array}$ \\
\hline
\end{tabular}


Diversidade e Gestão 5: 01-17. 2021

$$
\text { e-ISSN: 2527-0044 }
$$

http://costalima.ufrrj.br/index.php/diversidadeegestao

\begin{tabular}{|c|c|c|c|c|c|c|}
\hline & Masdevallia infracta Lindl. & HOLO & ENT & ANEC & NE & $\begin{array}{l}\text { ACR Cruz \& AF } \\
\text { Nunes-Freitas } \\
54\end{array}$ \\
\hline & $\begin{array}{l}\text { Polystachya concreta (Jacq.) Garay \& } \\
\text { Sweet }\end{array}$ & HOLO & ENT & ANEC & NE & $\begin{array}{l}\text { ACR Cruz \& NM } \\
\text { Correa } 112\end{array}$ \\
\hline & Polystachya sp1 & HOLO & ENT & ANEC & - & $\mathrm{NC}$ \\
\hline & Octomeria sp1 & HOLO & ENT & ANEC & - & $\mathrm{NC}$ \\
\hline \multirow{4}{*}{ Piperaceae } & Peperomia urocarpa Fisch. \& C.A.Mey. & HOLO & ANE & $\mathrm{ZOO}$ & NE & $\begin{array}{l}\text { ACR Cruz \& AF } \\
\text { Nunes-Freitas } \\
62\end{array}$ \\
\hline & Peperomia rotundifolia (L.) Kunth & HOLO & ANE & $\mathrm{ZOO}$ & NE & $\mathrm{NC}$ \\
\hline & Peperomia corcovadensis Gardner & HOLO & ANE & ZOO & LC & $\begin{array}{l}\text { ACR Cruz \& NM } \\
\text { Correa } 118\end{array}$ \\
\hline & Peperomia sp1 & HOLO & ENT & $\mathrm{ZOO}$ & - & $\begin{array}{l}\text { ACR Cruz et al. } \\
187\end{array}$ \\
\hline \multirow{9}{*}{ Polypodiaceae } & $\begin{array}{l}\text { Campyloneurum atlanticum R.C. Moran } \\
\text { \& Labiak }\end{array}$ & HOLO & - & ANEC & - & $\begin{array}{l}\text { ACR Cruz \& NM } \\
\text { Correa } 105\end{array}$ \\
\hline & Campyloneurum nitidum (Kaulf.) C.Presl & HOLO & - & ANEC & NE & $\begin{array}{l}\text { ACR Cruz \& AF } \\
\text { Nunes-Freitas } \\
66\end{array}$ \\
\hline & Campyloneurum rigidum Sm & HOLO & - & ANEC & NE & $\begin{array}{l}\text { ACR Cruz \& NM } \\
\text { Correa } 84\end{array}$ \\
\hline & $\begin{array}{l}\text { Microgramma crispata (Fée) R.M.Tryon \& } \\
\text { A.F.Tryon }\end{array}$ & HEM & - & ANEC & LC & $\begin{array}{l}\text { ACR Cruz \& AF } \\
\text { Nunes-Freitas } \\
60\end{array}$ \\
\hline & $\begin{array}{l}\text { Microgramma squamulosa (Kaulf.) de la } \\
\text { Sota }\end{array}$ & HOLO & - & ANEC & NE & $\begin{array}{l}\text { ACR Cruz \& NM } \\
\text { Correa } 132\end{array}$ \\
\hline & Microgramma tecta (Kaulf.) Alston & HEM & - & ANEC & NE & $\begin{array}{l}\text { ACR Cruz \& NM } \\
\text { Correa } 83\end{array}$ \\
\hline & $\begin{array}{l}\text { Microgramma vacciniifolia (Langsd. \& } \\
\text { Fisch.) Copel. }\end{array}$ & HEM & - & ANEC & NE & $\begin{array}{l}\text { ACR Cruz \& AF } \\
\text { Nunes-Freitas } \\
60\end{array}$ \\
\hline & Microgramma sp1 & HEM & - & ANEC & - & NC \\
\hline & Microgramma sp2 & HEM & - & ANEC & - & ACR Cruz \& AF \\
\hline
\end{tabular}


Diversidade e Gestão 5: 01-17. 2021

e-ISSN: 2527-0044

http://costalima.ufrrj.br/index.php/diversidadeegestao

\begin{tabular}{|c|c|c|c|c|c|c|}
\hline & & & & & & $\begin{array}{l}\text { Nunes-Freitas } \\
122\end{array}$ \\
\hline & Pecluma sicca (Lindm.) M.G.Price & HOLO & - & ANEC & NE & $\begin{array}{l}\text { ACR Cruz \& AF } \\
\text { Nunes-Freitas } \\
59\end{array}$ \\
\hline & Pecluma sp1 & HOLO & - & ANEC & - & $\begin{array}{l}\text { ACR Cruz \& NM } \\
\text { Correa } 138\end{array}$ \\
\hline & Phlebodium pseudoaureum J.Sm. & HOLO & - & ANEC & NE & $\begin{array}{l}\text { ACR Cruz \& NM } \\
\text { Correa } 134\end{array}$ \\
\hline & Pleopeltis astrolepis (Liebm.) E. Fourn. & HOLO & - & ANEC & NE & $\begin{array}{l}\text { ACR Cruz \& NM } \\
\text { Correa } 101\end{array}$ \\
\hline & Pleopeltis hirsutissima (Raddi) de la Sota & HOLO & - & ANEC & NE & $\begin{array}{l}\text { ACR Cruz \& AF } \\
\text { Nunes-Freitas } \\
125\end{array}$ \\
\hline & $\begin{array}{l}\text { Pleopeltis minima (Bory) J.Prado \& } \\
\text { R.Y.Hirai }\end{array}$ & HOLO & - & ANEC & NE & $\begin{array}{l}\text { ACR Cruz \& NM } \\
\text { Correa } 137\end{array}$ \\
\hline & Pleopeltis pleopeltifolia (Raddi) Alston & HOLO & - & ANEC & NE & $\begin{array}{l}\text { ACR Cruz \& AF } \\
\text { Nunes-Freitas } \\
61\end{array}$ \\
\hline & $\begin{array}{l}\text { Serpocaulon fraxinifolium (Jacq.) } \\
\text { A.R.Sm. }\end{array}$ & HOLO & - & ANEC & NE & $\begin{array}{l}\text { ACR Cruz \& AF } \\
\text { Nunes-Freitas } \\
123\end{array}$ \\
\hline & $\begin{array}{l}\text { Serpocaulon menisciifolium (Langsd. \& } \\
\text { Fisch.) A.R.Sm. }\end{array}$ & HOLO & - & ANEC & NE & $\begin{array}{l}\text { ACR Cruz \& AF } \\
\text { Nunes-Freitas } \\
67\end{array}$ \\
\hline & Adiantopsis radiata (L.) Fée & HOLO & - & ANEC & NE & $\begin{array}{l}\text { ACR Cruz \& AF } \\
\text { Nunes-Freitas } \\
55\end{array}$ \\
\hline Pteridaceae & Adiantum latifolium Lam. & EA & - & ANEC & NE & $\begin{array}{l}\text { ACR Cruz \& AF } \\
\text { Nunes-Freitas } \\
52\end{array}$ \\
\hline & Adiantum sp1 & EA & - & ANEC & - & NC \\
\hline Selaginellaceae & Selaginella muscosa Spring & EA & - & ANEC & NE & $\begin{array}{l}\text { ACR Cruz \& NM } \\
\text { Correa } 111\end{array}$ \\
\hline
\end{tabular}


Em relação às síndromes de dispersão de esporos e sementes, 56 espécies são anemocóricas (65,9\%), 27 são zoocóricas $(35,8 \%)$ e apenas duas autocóricas $(2,3 \%)$ (Figura 2 ). Nossos resultados corroboram com diversos autores (Gentry \& Dodson 1987, Benzing 1990, Mondragón et al. 2015) e ressalta-se que a anemocoria pode ocorrer de duas formas: através de diásporos diminutos, em orquídeas, samambaias e licófitas, e por sementes um pouco maiores com estruturas plumosas, como ocorrem em bromélias dos gêneros Tillandsia e Vriesea (Gentry \& Dodson 1987). Apesar de a maioria das sementes tenderem a cair próximas a plantamãe, estudos genéticos indicam que há dispersão a média e longa distâncias em epífitas (Trapnell et al. 2004, Ramírez-Padilla 2008). A possibilidade de colonização de novos habitats livres de competição e da alta densidade é uma das principais vantagens da dispersão a longas distâncias (Howe \& Smallwood 1982) e as alternativas mais vantajosas para epífitas são produção de grande quantidade de sementes e o uso de dispersores que levem os diásporos a microhabitats específicos (Hughes et al. 1994), como ocorre em Cactaceae (Guaraldo et al. 2013), Araceae, Piperaceae e alguns gêneros de Bromeliaceae (Vander \& Longland 2004). Os padrões de dispersão das epífitas, juntamente com a tendência de propagação clonal de algumas das principais famílias, explicam a distribuição irregular que caracteriza a comunidade e influenciam a diversidade genética das populações (Mondragón et al. 2015). A dispersão de diásporos a longas distâncias são fundamentais na manutenção do fluxo gênico e favorecem a formação de novas populações (Bernal 2006, Mondragón et al. 2015).

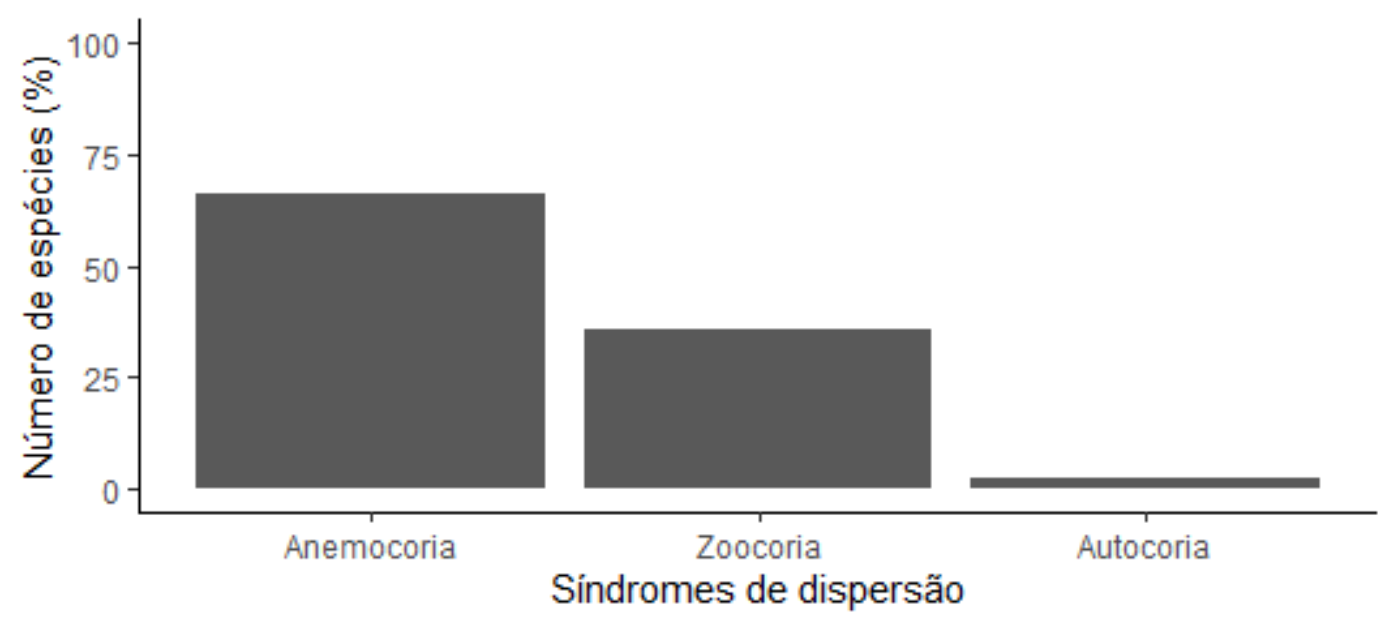

Figura 2. Proporção do número de espécies (\%) de epífitas vasculares por síndromes de dispersão de diásporos da Vila Dois Rios, Ilha Grande, Angra dos Reis, RJ. 
A maioria das epífitas da Vila Dois Rios é endêmica da Floresta Atlântica (39 espécies, 60,9\%). Quanto à distribuição geográfica nas regiões geopolíticas do país, a maior parte $(38 ; 59,4 \%)$ é amplamente distribuída e $22(34,4 \%)$ ocorrem em apenas duas regiões (Figura 3). Dezoito espécies $(28,1 \%)$ ocorrem somente nos estados do Sudeste e Sul e quatro $(6,3 \%)$ delas do Sudeste e Nordeste. Três espécies são restritas ao Sudeste (4,6\%): uma Cactaceae, Rhipsalis clavata F.A.C.Weber, e duas Bromeliaceae, Canistropsis microps (E.Morren ex Mez) Leme e Neoregelia johannis (Carrière) L.B.Sm. Estas duas bromélias ocorrem exclusivamente nos estados de São Paulo e Rio de Janeiro. O Domínio Atlântico apresenta as condições ideais para a ocorrência de epífitas, com alta umidade atmosférica, temperatura e precipitação, além de oferecer alta heterogeneidade de habitats (Leitman et al. 2015). Segundo MeniniNeto et al. (2016), as áreas litorâneas e montanhosas das Regiões Sul e Sudeste do Brasil, bem como o litoral sul da Bahia, se destacam dentro da Floresta Atlântica devido às acentuadas taxas de riqueza e endemismos, o que justifica os resultados obtidos. Duas espécies não são nativas do Brasil, uma é a holoepífita Tradescantia zebrina Heynh. ex Bosse, que é naturalizada, e a outra é a hemiepífita Syngonium podophyllum Schott, considerada exótica, ambas ocorreram tanto na área urbana quanto no interior das florestas.

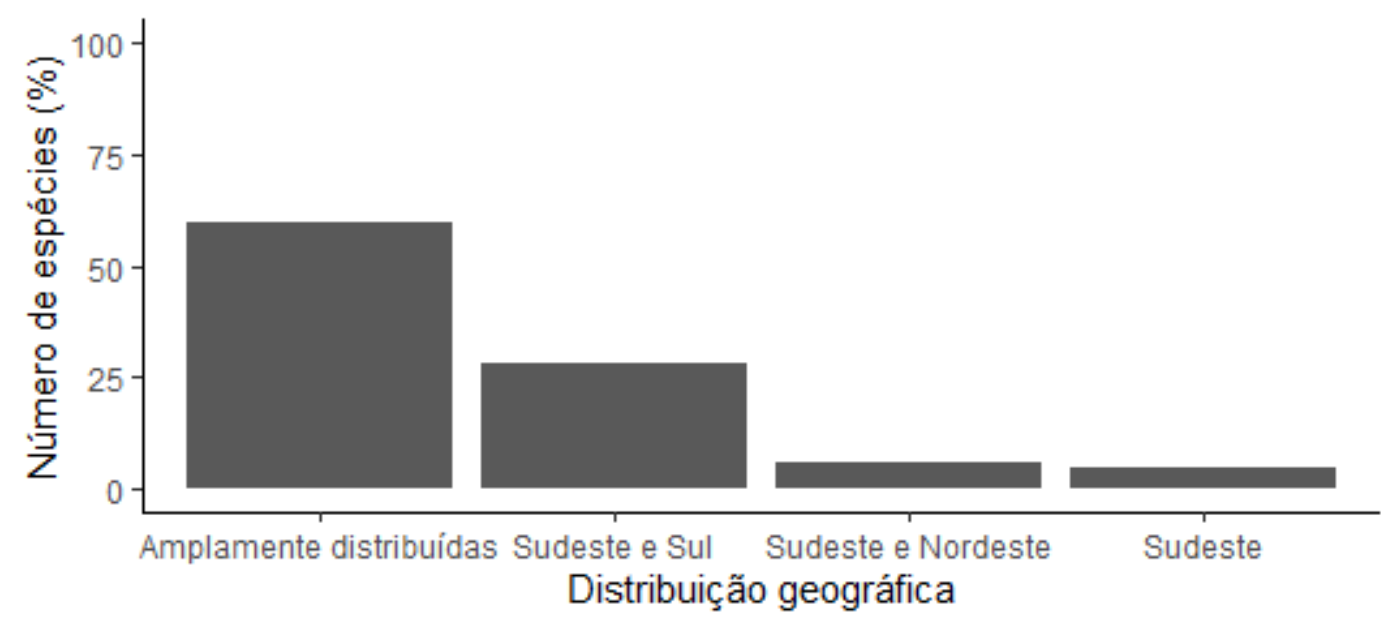

Figura 3. Distribuição geográfica das espécies de epífitas vasculares da Vila Dois Rios, Ilha Grande, Angra dos Reis, RJ.

Apenas $26,6 \%$ das epífitas apresentaram estudos suficientes que permitem classificá-las quanto ao grau de ameaça de extinção pelo Centro Nacional de Conservação da Flora (2020) e se encontram avaliadas quanto ao estado de conservação, estas estão categorizadas em situação pouco preocupante ( $L C=17$ espécies), pois são abundantes $\mathrm{e}$ amplamente distribuídas. A maioria das espécies possui estado de conservação desconhecido ( $N E=47$ espécies; 73,4\%) já que sequer 
foram avaliadas. Ressaltamos a importância deste resultado, pois as espécies registradas podem estar sob algum grau de ameaça e devem ser priorizadas em estudos ecológicos populacionais futuros. Segundo Ramos et al. (2021), as principais ameaças às epífitas são redução das áreas de floresta, perda de polinizadores e dispersores e pressões de coleta causada pelo extrativismo ilegal. E, além destes fatores, ainda há os efeitos causados pela fragmentação dos habitats, uma vez que comunidades de bordas de florestas ou em árvores isoladas tendem a apresentar menor diversidade do que aquelas no interior de florestas maduras (Amici et al. 2019, Ramos et al. 2021).

\section{Conclusão}

O presente estudo é o primeiro levantamento de epífitas vasculares realizado em área urbana e florestas secundárias da Vila Dois Rios, Ilha Grande. Nossos resultados mostram que há elevada riqueza de espécies e reforçam o importante papel da arborização urbana para a conservação. As árvores das praças e avenidas da vila devem ser mantidas e protegidas, especialmente as mais velhas e de grande porte, uma vez que estas abrigam uma parcela fundamental da biodiversidade epifítica e podem servir como pontes para o fluxo gênico das populações. Adicionalmente, reforçamos a relevância ecológica desta comunidade como recurso alimentar para a fauna por meio de processos relacionados à polinização e dispersão mesmo em ambientes antropizados. Ressaltamos ainda a ocorrência de espécies endêmicas da Floresta Atlântica e da Região Sudeste do país, associado ao fato da maior parte delas não terem sido avaliadas quanto ao grau de ameaça de extinção e precisam ser priorizadas em pesquisas ecológicas populacionais futuras. Por fim, a Ilha Grande pode ser considerada um local de conservação da biodiversidade do Rio de Janeiro, principalmente de epífitas. Enfatizamos a necessidade da realização de mais pesquisas sobre os padrões e processos que envolvem a comunidade epifítica de forma que possam subsidiar a elaboração de estratégias de conservação eficientes das espécies em área urbana e em ecossistemas naturais.

\section{Agradecimentos}

A Coordenação de Aperfeiçoamento de Pessoal de Nível Superior, a Universidade Federal do Rio de Janeiro, ao Museu Nacional, ao Programa de Pós-graduação em Ciências Biológicas (Botânica), ao Instituto Estadual do Ambiente, ao Parque Estadual da Ilha Grande, ao Centro de Estudos Ambientais e Desenvolvimento Sustentável da Universidade do Estado do Rio de Janeiro e todos os pesquisadores que contribuíram direta ou indiretamente na realização dessa pesquisa, especialmente aos taxonomistas Dr. Marcus A. N. Coelho, MSc. George A. de Queiroz, Dr. Diego R. Gonzaga e Dr. Felipe Fajardo V. A. Barberena.

\section{Referências}

Adhikari YP, Fischer HS, Fischer A (2012) Host tree utilization by epiphytic orchids in different land-use intensities in Kathmandu valley, Nepal. Plant Ecology 213: 13931412. 
Ackerman J D (1986) Coping with the epiphytic existence: pollination strategies. Selbyana 9: 52-60.

Amici AA, Nadkarni NM, Williams CB, Gotsch SG (2020) Differences in epiphyte biomass and metabolism composition along landscape and within- crown spatial scales. Biotropica 52: 46-58.

Alves MEO, Brun C, Forno RSD, Essi L (2014) Levantamento de espécies epífitas vasculares da zona urbana do município de Palmeira das Missões, RS, Brasil. Ciência e Natura 36(3): 268-276.

Araújo DSD, Oliveira RR (1988) Reserva Biológica da Praia do Sul (Ilha Grande, Estado do Rio de Janeiro): lista preliminar da Flora. Acta Botanica Brasilica 1(2, Suppl. 1), 83-94.

Bastos, MP, Callado CH (2009) O ambiente da Ilha Grande. Rio de Janeiro: UERJ / CEADS. 562 p.

Bataghin FA, Pires JSR, Barros F, Muller A (2017) Epífitas vasculares da Estação Ecológica Barreiro Rico, Anhembi, SP, Brasil: diversidade, abundância e estratificação vertical. Hoehnea [online] 44: 172-183.

Benzing DH (1990) Vascular epiphytes. Cambridge: Cambridge University Press. 354 p.

Bernal R (2006) Estudio Metapoblacional de Tillandsia recurvata L. en el Valle de Tehuacán, Puebla [Metapopulation Study of Tillandsia recurvata L. in the Tehuacan Valley, Puebla]. Ph.D. Thesis. Universidad Nacional Autónoma de México, Mexico City.

Brazil Flora Group (2021): Brazilian Flora 2020 project - Projeto Flora do Brasil 2020. v393.274. Instituto de Pesquisas Jardim Botanico do Rio de Janeiro. Dataset/Checklist. doi:10.15468/1mtkaw

Callado CH, Barros AAM, Ribas LA, Albarello N, Gagliardi R, Jascone CE (2009) Flora e Cobertura vegetal. In: Bastos MP, Callado $\mathrm{CH}$. O ambiente da Ilha Grande. Rio de Janeiro: UERJ / CEADS. pp. 562.

Centro Nacional de Conservação da Flora (2020) CNCFlora. Disponível: http://cncflora.jbrj.gov.br/portal. Acessado em 18 de março de 2020.

Cruz ACR, Nunes-Freitas AF (2017) Diversidade e conservação das epífitas vasculares da Ilha Grande, RJ. Anais do $6^{\circ}$ Simpósio de Gestão Ambiental e Biodiversidade. Disponível: http://itr.ufrrj.br/sigabi/anais. Acessado em 21 de outubro de 2019.

Cruz ACR, Nunes-Freitas AF (2019) Epífitas vasculares da mata de restinga da Praia do Sul, Ilha Grande, RJ, Brasil. Rodriguésia [online] 70: e03192017. Disponível: http://dx.doi.org/10.1590/2175-7860201970047. Acessado em 09 de novembro de 2019.

Devens KU, Geraldini APB, Amadeo RM, Caxambu MG, Magnoni PHJ (2015) Levantamento de epífitas na arborização urbana do município de Luiziana - Paraná. RevsBau 10(4): 1-11.

Dettke GA, Orfrini AC, Milaneze-Gutierre MA (2008) Composição florística e distribuição de epífitas vasculares em um remanescente alterado de floresta estacional semidecidual no Paraná, Brasil. Rodriguésia 59(4): 859-872.

Dislich R, Mantovani W (1998) A flora de epífitas vasculares da reserva da Cidade Universitária "Armando de Salles Oliveira" (São Paulo, Brasil). Boletim de Botânica da Universidade de São Paulo 17: 1-83.

Flora do Brasil 2020 em Construção (2020) Instituto de Pesquisas Jardim Botânico do Rio de Janeiro. Disponível: http://floradobrasil.jbrj.gov.br/. Acessado em 17 de março de 2020.

Furtado SG, Neto LM (2015) Diversity of vascular epiphytes in urban environment: a case study in a biodiversity hotspot, the Brazilian Atlantic Forest. Ces Revista 29(2): 82101.

Gama SVG, Silva LGAE, Salgado CM (2009) Geologia, relevo e solos. In: Bastos MP, Callado $\mathrm{CH}$, Orgs. O ambiente da Ilha Grande. Rio de Janeiro: UERJ / CEADS. pp. 562.

Gentry AH, Dodson CH (1987) Diversity and biogeography of neotropical vascular epiphytes. Annals of the Missouri Botanical Garden 74: 205-233. 
Guaraldo AC, Boeni BO, Pizo MA (2013) Specialized seed dispersal in epiphytic cacti and convergence with mistletoes. Biotropica 45: 465-473.

Guzmán-Marín R, Saldaña A (2017) Contribución del epifitismo accidental a la distribución de especies de plantas vasculares en un bosque templado lluvioso. Gayana. Botánica 74: 226-228.

Hietz P (1998) Diversity and conservation of epiphytes in a changing environment. International Union of Pure and Applied Chemistry 70: 11.

Hietz P (2005) Conservation of vascular epiphyte diversity in mexican coffee plantations. Conservation Biology 19 (2): 391-399.

Holwerda F, Bruijnzeel LA, Barradas VL, Cervantes J (2013) The water and energy etaboli of a shaded coffee plantation in the lower montane cloud forest zone of central Veracruz, Mexico. Agricultural and Forest Meteorology 173: 1-13.

Howe HF, Smallwood J (1982) Ecology of seed dispersal. Annual Review of Ecology and Systematics 13: 201-228.

Hughes L, Dunlop M, French K, Leishman MR, Rice B, Rodgerson L, Westoby M (1994) Predicting dispersal spectra: a minimal set of hypotheses based on plant attributes. Journal of Ecology 82: 933-950.

INEA - Instituto Estadual do Ambiente (2011) Parque Estadual da Ilha Grande: plano de manejo (fase 2). Resumo executivo. Instituto Estadual do Ambiente, Rio de Janeiro. $98 \mathrm{p}$.

Leitman P, Amorim AM, Sansevero JBB, Forzza RC (2015) Floristic patterns of epiphytes in the Brazilian Atlantic Forest, a biodiversity hotspot. Botanical Journal of the Linnean Society 179: 587-601.

Madison M (1977) Vascular epiphytes: their systematic occurrence and salient features. Selbyana 2 (1): 1-13.

Magnusson WE, Lima AP, Luizão RC, Luizão F, Costa FRC, Castilho CV, Kinupp VF (2005) RAPELD: a modification of the Gentry method for biodiversity surveys in long-term ecological research sites. Biota Neotropica 5(2).

Menini Neto L, Furtado SG, Zappi DC, Oliveira-Filho AT, Forzza RC (2016) Biogeography of epiphytic Angiosperms in the Brazilian Atlantic forest, a world biodiversity hotspot. Rev Bras Bot 39:261-273

Nieder J, Engwald S, Barthlott W (1999) Patterns of neotropical epiphyte diversity. Selbyana 20: 66-75.

Nieder J, Engwald S, Klawu M, Barthlott W (2000) Spatial distribution of vascular epiphytes (including hemiepiphytes) in a lowland Amazonian rain forest (Surumoni crane plot) of southern Venezuela. Biotropica 32: 385-396.

Nieder J, Prosperi J, Michaloud G (2001) Epiphytes and their contribution to canopy diversity. Plant Ecology 153: 51-63.

Nunes-Freitas AF, Rocha-Pessôa TC, Dias AS, Ariani CV, Rocha CFD (2009) Bromeliaceae da Ilha Grande, RJ: revisão da lista de espécies. Biota Neotropica 9(2), 213-219.

Oliveira RR, Coelho Netto AL (2000) Processos interativos homem-floresta na evolução da paisagem da Ilha Grande, RJ. Revista do Departamento de Geografia UERJ 8: 2938.

Padilha TP, Santos JRR, Custódio SZ, Oliveira LC, Santos R, Citadini-Zanette V (2015) Comunidade epifítica vascular do Parque Estadual da Serra Furada, sul de Santa Catarina. Ciência e Natura 37: 64-78.

Padmawathe R, Qureshi Q, Rawat GS (2004) Effects of selective logging on vascular epiphyte diversity in moist lowland forest of Eastern Himalaya, India. Biological Conservation 119: 81-92.

Ramírez-Padilla CA (2008) Análisis de la Dispersión de Semillas en una Metapoblación de la epífita Tillandsia recurvata L. (Bromeliaceae) a Través del Uso de Microsatélites. [Analysis of seed dispersal in a metapopulation of Tillandsia recurvata L. (Bromeliaceae) through the use of microsatellites]. M.Sc. Thesis. Universidad Nacional Autónoma de México, Mexico City.

Ramos, FN et al. (2019) ATLANTIC EPIPHYTES: a data set of vascular and non-vascular epiphyte plants and lichens from the Atlantic Forest. Ecology 100(2). 
Ramos FN, Montara SR, Elias, JPC (2021) Vascular Epiphytes of the Atlantic Forest: Diversity and Community Ecology. In: Marques MCM, Grelle CEV, Editors. The Atlantic Forest. Springer. pp. 133-146.

Richards JH, Luna IMT, Waller DM (2020) Tree longevity drives conservation value of shade coffee farms for vascular epiphytes. Agriculture, Ecosystems \& Environment 301(1).

Rocha CFD, Bergallo HG, Alves MAS, Van Sluys M (2003) A biodiversidade nos grandes remanescentes florestais do Estado do Rio de Janeiro e nas restingas da Mata Atlântica. São Carlos: RiMa Editora.

Staudt MG, Lippert APU, Cunha S, Becker DFP, Marchioretto MS, Schmitt JL (2012) Composição florística de epífitos vasculares do Parque Natural Municipal Tupancy, Arroio do Sal, RS, Brasil. Pesquisas, Botânica 63: 177-188.

Trapnell DW, Hamrick JL, Nason JD (2004) Three-dimensional fine-scale genetic structure of the neotropical epiphytic orchid, Laelia rubescens. Molecular Ecology 13: 11111118.

Vander SB, Longland WS (2004) Diplochory: are two seed dispersers better than one? Trends in Ecology and Evolution 19: 155-161.

Zotz G (2016) Plants on plants - the biology of vascular epiphytes. Cham, Springer International Publishing. 282 p. 